\title{
Modelos y prácticas de educación y comunicación: una perspectiva sociocultural*
}

Raúl Fuentes Navarro ${ }^{10}$

\section{Resumen}

En este artículo se argumenta en favor de una reconceptualización de las relaciones entre las prácticas educativas y las comunicativas, basada en una perspectiva sociocultural, es decir, una que permite investigar las relaciones entre la acción socialmente estructurada y el sentido generado por los sujetos. Desde ahí, y a propósito de las oportunidades que abre la irrupción de Internet en todas las interacciones socioculturales, se sugiere una metodología comunicacional que permita evitar el reduccionismo en la investigación tanto de la educación y la comunicación como de sus relaciones.

\section{Palabras clave}

Comunicación, educación, Internet, estudios socioculturales

\section{Summary}

The aim of this article is to foster a critical reconceptualization of the relationship between education and communication practices. Its proposal of a sociocultural approach is intended to sustain research on the relations between structured social action and subjectively generated meaning. Following this proposal, and focusing on the cognitive opportunities provided by the irruption of Internet in every sociocultural interaction, a communication methodology is suggested, as a way to avoid reductionism in research on communication, on education, and on its relationships.

\section{Keywords}

Communication, education, Internet, sociocultural studies

Hace varias décadas que, especialmente en América Latina, se intuyen y se exploran las múltiples confluencias aparentes entre la comunicación y la educación, desde diversos planos de la práctica social y para distintos propósitos. Bajo diferentes denominaciones, estas intersecciones han sido exploradas de muchas maneras, no siempre coincidentes. En este trabajo propongo un abordaje que de alguna manera parte de la categoría "educomunicación", propuesta por el colega brasileño Ismar de Oliveira Soares (1999) para referirnos a "toda acción comunicativa en espacios educativos, realizada con el objetivo de producir o desarrollar ecosistemas comunicativos", pues desde esta perspectiva el ambiente aparece como resultante de las interacciones y tiene en el fondo

\footnotetext{
- Artículo recibido el 12 de abril de 2004 y arbitrado el 15 de abril de 2004.

${ }^{10}$ Profesor-investigador Numerario, Departamento de Estudios Socioculturales, ITESO, Guadalajara, Jal. , México, E-mail: raul@iteso.mx 
un modelo sociocultural centrado en la producción de sentido por sujetos concretamente situados. Sin embargo, como se verá, sigue siendo necesaria la discusión en el plano conceptual y la búsqueda de mejores términos para abordar las múltiples cuestiones involucradas.

Comienzo por cuestionar los fundamentos de una pregunta cuya respuesta ha sido muy problemática, en la teoría y en la práctica, durante al menos cuatro décadas en América Latina: la que considera que la educación y la comunicación son dos entidades, ámbitos o tipos de acción diferentes que hay que encontrar cómo unir. Ante las dificultades de un consenso claro en ese sentido, conviene reconocer que el problema radica, sobre todo, en la historia de la institucionalización de dos campos académicos con tendencias comunes a la disciplinarización y con una autonomía científica muy escasa, debido sobre todo al peso que sobre el cultivo del conocimiento en ambos casos han ejercido las instituciones sociales con las que han identificado sus estudios.

Esto nos lleva a una discusión más precisa que la escisión entre la teoría y la práctica o los efectos de las tecnologías, nuevas o no. Y nos permite salir de la tramposa disyuntiva de si la comunicación debe formar parte de la educación o viceversa. Finalmente, nos ayuda a superar pugnas cotidianas como las que enfrentan a los "representantes" de los medios con los de la escuela y a través de estas referencias a los "defensores" de la cultura audiovisual con los de la cultura letrada, o hasta a los "posmodernos" con los "modernizadores".

No puede haber duda de que, históricamente, la institucionalización de la educación como un campo de práctica social y como un campo de saber académico especializado es anterior, por mucho, a la institucionalización de la comunicación en esos dos ámbitos. Cuando, entre las dos guerras mundiales del siglo pasado, en Estados Unidos se fueron tomando decisiones para institucionalizar los estudios de comunicación en las universidades, había tantos antecedentes académicos para hacerlo en relación con la educación, en el campo de las humanidades, como con la propaganda, en el de las ciencias sociales. Esa disyuntiva es crucial para entender la historia de las llamadas "Ciencias de la Comunicación", que hubieran podido ser "hermanas" de las "Ciencias de la Educación".

Pero los proyectos fundacionales del estudio de la comunicación, por diversas razones, se resolvieron en términos del avance en el conocimiento y control de los mecanismos propagandísticos, centrados en la difusión masiva y selectiva de mensajes persuasivos, y no de los educativos, asociados a la construcción democrática de comunidades de conocimiento y acción; y al conseguirse no sólo los apoyos políticos y financieros, sino también la legitimidad académica de la investigación con ese sesgo, la separación entre comunicación y educación y la escisión entre humanidades y ciencias sociales quedó consagrada y el modelo de la Mass Communication consolidado, independientemente de su consistencia epistemológica, primero en Estados Unidos y luego en el resto del mundo, a donde se exportó en el contexto de la modernización y de la guerra fría. Timothy Glander, que documentó sistemáticamente esta historia, parte de que:

La educación y la comunicación están fundamentalmente vinculadas, inescapablemente afiliadas en la teoría y en la práctica. Los filósofos de la educación, de Sócrates a Dewey y Freire, lo han reconocido así y han tratado de clarificar esta relación. La educación y la comunicación no pueden ser separadas, aunque nuestras disposiciones académicas presentes hagan creer que pueden ser segregadas. La organización contemporánea del conocimiento sugiere que educación y comunicación 
son fenómenos distintos, que pueden ser estudiados y practicados en aislamiento mutuo. (...) esta división (...) afectó [tanto a] la emergencia y crecimiento del nuevo campo de la comunicación [como a] los asuntos educativos en el siglo XX (Glander, 2000: $x)$.

Durante muchos años, por ende, la idea central que impulsó las búsquedas articuladoras de la comunicación y la educación fue la del desarrollo, la de la modernización, horizonte de política social que concebía instrumentalmente a la educación como un medio, como un factor determinante del progreso individual o colectivo y también, posteriormente, a la comunicación como un instrumento que podría servir a la extensión de los "servicios" educativos. Sobre todo desde que la televisión comenzó a extenderse, hace ya más de medio siglo, y se retomaron y amplificaron con ella las promesas y experiencias suscitadas por ciertos usos educativos de la radio o del cine, se ha hablado tanto del "potencial educativo" de los medios de difusión masiva como de la "esencia comunicativa" de los procesos escolares. Creo que debemos cambiar de perspectiva.

Pero es un hecho que las concepciones instrumentales, en este caso de la comunicación y la educación, están lejos de haber desaparecido o de haber sido "superadas" en los discursos políticos o en los imaginarios sociales del siglo XXI. Ahora, más que nunca, el eje tecnológico parece articular globalmente las representaciones de la sociedad posible y deseable, se le llame "sociedad del conocimiento", "de la información" o como se quiera. La obra del norteamericano Dan Schiller (1999), por ejemplo, nos permite avanzar en el desentrañamiento crítico de este "Capitalismo Digital" o informacional en que estamos inmersos, aunque es indispensable construir las implicaciones que esto tiene en el plano sociocultural, como lo hace ejemplarmente Jesús Martín Barbero (2002a). Para muchos analistas está claro que los sistemas educativos han sido "rebasados" por los sistemas de comunicación, y probablemente haya mucho de verdad en ese diagnóstico, aunque cada vez es menos claro por qué y qué márgenes de maniobra quedan, en términos de proyecto social, además de la definitiva "informatización de la sociedad".

Desde una concepción instrumental, me parece, no hay mucho que discutir, pues las tendencias diseñadas estratégicamente reducen las alternativas. Para avanzar en la discusión es necesario, de entrada, contrarrestar el reduccionismo que identifica comunicación con medios de difusión o, peor, con aplicaciones tecnológicas, y a la educación con lo que sucede únicamente en las escuelas. Y para ello, es necesaria una clarificación conceptual que permita no confundir niveles y ámbitos de relación. Sobre todo, que facilite la comprensión del lugar que ocupan los factores tecnológicos en procesos esencialmente humanos, intersubjetivos, socioculturales, como la educación y la comunicación. La expansión de la Internet en la última década nos ofrece una oportunidad única para hacer esto, que habrá que aprovechar.

Se pueden proponer tres ámbitos en los cuales puede ubicarse y formularse la convergencia de la educación y la comunicación. Uno, el más abstracto, es el de los conceptos que sirven para delimitar aspectos de la realidad sociocultural vivida y orientar su entendimiento y apropiación. Otro es el ámbito de las instituciones, el de las configuraciones que la sociedad impone a los individuos y las colectividades para estructurar sus acciones en el tiempo y el espacio. El tercer ámbito, quizá el más importante, pero difícil de explicar al margen de los otros dos, es el de las prácticas cotidianas, el de las acciones y las interacciones situadas, el de los proyectos que los sujetos impulsan y confrontan para constituir sus identidades y hacerlas prevalecer socialmente. En los tres ámbitos es necesaria la inversión articulada de esfuerzos de investigación, y la perspectiva sociocultural, es decir, una que permite investigar las 
relaciones entre instituciones, discursos y prácticas, o dicho de otro modo, entre la acción socialmente estructurada y el sentido generado por los sujetos, facilita la formulación de marcos pertinentes y productivos, tanto académica como socialmente.

Desde esta concepción, comunicación y educación son nombres genéricos para proyectos, o tipos de proyectos socioculturales, en los que los sujetos creen y por los que luchan en la práctica cotidiana. El educar o hacer emerger del sujeto las manifestaciones sociales de su autotrascendencia, y el comunicar o poner en común los significados y el sentido de lo que sucede en el entorno, son acciones necesariamente intersubjetivas. Nadie se educa sólo, nadie se comunica consigo mismo. Ambas acciones son también, por ello, vectores primordiales de la socialización y de la construcción social de las identidades de los sujetos. Comunicación y educación son procesos dialécticos, mediante los cuales al mismo tiempo se estructuran los individuos, las comunidades y la sociedad que los contiene. En gran medida, son procesos simbólicos, mediados primariamente por el lenguaje, constitutivos básicos de las tramas culturales que les dan forma específica, desde un tiempo y un lugar determinados, a las relaciones del hombre con el mundo.

En ese sentido elemental, la educación y la comunicación son los mecanismos sociales por los que se genera y reproduce constantemente la cultura, sistema de sistemas de significación y valoración convencionalmente adoptados para interpretar la vida en todas sus dimensiones. Son también, desde el punto de vista del individuo, los mecanismos que hacen posible socialmente la operación de los procesos de asimilación y acomodación que Piaget (1971) postulaba como constitutivos de los equilibrios dinámicos de la inteligencia. En este plano, en el que confluyen conceptualmente la génesis de la estructuración social, de la cultura, del lenguaje, de la identidad y de la inteligencia no hay en realidad problema alguno para fundamentar una sólida e inseparable relación dialéctica entre comunicación y educación, entre cultura y aprendizaje, entre socialización e individuación. Las dificultades comienzan cuando se introducen en este esquema abstracto las consideraciones históricas, es decir, las densas y complejas configuraciones institucionales que las sociedades concretas han impuesto en el tiempo y en el espacio a procesos tan importantes para su reproducción como la comunicación y la educación.

Hay al menos dos condiciones ineludibles al pasar a este plano: la educación y la comunicación no son procesos abstractos que realicen individuos ideales, sino prácticas específicas de sujetos concretos, concretamente socializados, y la estructura social consiste en un sistema de posiciones desniveladas y diferenciadas. Además, ni la educación ni la comunicación son procesos espontáneos, ajenos a la normatividad social; por su propia importancia, hace mucho que están institucionalizados. Muchos de los discursos sobre las relaciones entre comunicación y educación caen en esta doble trampa: reducen la educación a la escuela y la comunicación, a los medios de difusión, y plantean sus relaciones como si las instituciones y las personas que las habitan fueran entidades ideales. Y, como es obvio, no existen ni pueden existir instituciones educativas o comunicativas, o personas que operen unas u otras, que actúen en abstracto. De ahí que los esquemas simples del tipo enseñanza-contenido-aprendizaje no puedan hacerse pertinentemente equivalentes a los del tipo emisión-mensaje-recepción. Si esos esquemas para algo sirven, no es ciertamente para comprender las relaciones entre educación y comunicación, a no ser que éstas se reduzcan a procesos institucionalizados, mecánicos y lineales, de difusión de información.

Cuando se piensa en la educación como la "enseñanza de contenidos" o en la comunicación como la "transmisión de mensajes", lo que se representa es el esquema mecánico y burocrático, impersonalizado, que la configuración institucional predominante 
ha impuesto a estos procesos socioculturales. Es pensar el mundo sin sujetos, es reducir a los individuos a engranajes de una maquinaria en la que sólo deben hacer lo que se les ordena, asimilar lo que se les impone, responder a lo que se les pregunta y acatar ese estado de cosas como si fuera natural, o al menos inevitable. Según la más elemental lógica, entre las ideas abstractas de lo que es la educación o la comunicación o, peor, de lo que deben ser, y esos esquemas reduccionistas no hay la mínima consistencia. ¿Será la vieja distancia entre la teoría y la práctica o será que utilizamos un modelo inadecuado para construir la relación entre ambas?

Cambiemos el sentido del esquema, aunque sea sin complejizarlo demasiado. A la idea de que la enseñanza de contenidos o la transmisión de mensajes son los núcleos centrales de la acción educativa o de la acción comunicativa, opongamos la idea de que el aprendizaje de métodos y la interpretación de discursos son lo primordial. Es como cambiar el concepto de un mercado dominado por la oferta por un mercado dominado por el consumo, donde el cliente, y no el productor, es el que "manda". Cuando se trata de la valorización económica de mercancías, el esquema puede ser muy útil, pero ni siquiera en el comercio de productos materiales las relaciones son tan simples. Sin embargo, pensar en que el "destinatario" de las acciones educativas o comunicativas es un sujeto capaz de valorar lo que necesita y de controlar responsablemente la interacción que supone el obtenerlo, especialmente si se trata de un conjunto de competencias simbólicas, ayuda a reconocer la complejidad de los sistemas involucrados, tanto dentro como fuera de las instituciones formales especializadas.

Así, ciertos principios epistemológicos constructivistas, emergentes en la ciencia social contemporánea y opuestos a los postulados funcionalistas todavía prevalecientes, proponen, primero, considerar a los seres humanos como entes cognitivamente autónomos; segundo, como practicantes reflexivos de la comunicación con otros, y tercero, como interventores moralmente responsables, si no es que creadores, de las mismas realidades sociales en las cuales acaban viviendo (Krippendorff, 1993). En otras palabras, si los analistas y operadores de la educación y la comunicación institucionalizadas se asumen como agentes socioculturales, esto es, en la práctica, como intérpretes de lo que los sujetos destinatarios hacen cuando generan aprendizaje y cuando producen sentido, podrán de una mejor manera reorientar y reenfocar los recursos de que disponen para que la enseñanza y la emisión faciliten el aprendizaje y la interpretación más pertinentes y significativos.

De lo que se trata, en términos simples, es de impulsar una estrategia de readecuación de esquemas conceptuales, que deben complejizarse y flexibilizarse si se busca comprender y aprovechar la convergencia de la educación y de la comunicación en la práctica. Esa convergencia no puede estar ni en las instituciones, ni en el entorno social, ni en el equipamiento tecnológico: está en los sujetos que interactúan o no está en ninguna parte, excepto quizá en los deseos o en los modelos teóricos más abstractos.

Hablando en términos que pueden facilitar esta convergencia conceptual, la construcción de "competencias para el aprendizaje" como eje de los procesos educativos supone un modelo centrado en ciertas modalidades específicas de relación de los sujetos humanos con el conocimiento, en las cuales se constituyen y transforman mutuamente los sujetos y sus objetos de conocimiento. Pero el "conocimiento", desde este punto de vista, es una trama de esquemas o sistemas de significación socialmente construidos y subjetivamente incorporados como estructuras complejas de representaciones cognoscitivas de aspectos significativos de la realidad. Más que los "contenidos" específicos de esas representaciones, importa destacar las reglas y esquemas generativos de la significación 
y de la valoración de esos contenidos. El conjunto de estas reglas y esquemas generativos constituye la cultura y el equilibrio dinámico de sus incorporaciones individuales, la inteligencia. Más aún, como argumentaré más adelante, estas estructuras de saberes compartidos se convierten en modelos de la realidad o, si se quiere, en la realidad misma, que tiende a no poderse ver de otra manera.

Si esto es interpretable así, y propuestas como la de la "ecología cognitiva", de Pierre Levy (1993), lo sustentan y desarrollan muy estimulantemente, el conocimiento apropiado por el sujeto define las competencias operativas específicas de cada individuo para interpretar su entorno y dirigir la acción sobre él. Estas competencias están socialmente determinadas y por lo tanto son el objeto de transformación de las prácticas educativas institucionalizadas. Los esquemas cognoscitivos que la escuela enseña a sus estudiantes tienen siempre referencias y recortes específicos, sea por efecto del programa, del nivel, del contexto, de la ideología institucional o del empleo de más o menos y mejores o peores recursos didácticos, pero también, y esto es lo que suele descuidarse más, el sujeto que aprende cuenta además, desde muy pequeño, con esquemas cognoscitivos específicos que no provienen de la enseñanza escolarizada, pero que igualmente intervienen, muchas veces más decisivamente, en la construcción y reconstrucción constantes de las competencias culturales con las que el sujeto se integra al mundo en el que vive. Jesús Martín Barbero ha clarificado esta relación crecientemente contradictoria entre "el ecosistema comunicativo que constituye el entorno educacional difuso $y$ descentrado en que estamos inmersos" (2002b: 332).

Y aquí es donde debe reconectarse productivamente, desde esta perspectiva sociocultural, la acción educativa institucionalizada con las acciones comunicativas igualmente institucionalizadas, es decir, con la presencia cada día más omniabarcante de la acción de los medios de difusión masiva. Lo que los medios hacen socialmente no es producto sólo de las tecnologías que utilizan, sino sobre todo de las formas en que intervienen sobre los sistemas culturales, es decir, sobre las reglas y esquemas generativos de la significación y de la valoración intersubjetivas del entorno, sobre las competencias necesarias para conocer e interpretar el mundo, incluyendo las competencias de uso de las tecnologías, para producir sentido. Y aquí es donde se encuentran las principales divergencias entre las prácticas de las instituciones educativas y las prácticas de las instituciones comunicativas, ninguna de las cuales opera ni mecánica ni linealmente. No hablamos de flujos de información, sino de formaciones culturales. Es por ello que la realidad comunicativa actual, caracterizada por un creciente protagonismo de los medios y tecnologías de información en la producción y circulación discursiva, por una explosión de pedagogías para la generación, transmisión y asimilación de conocimiento, y por una acelerada hibridación de las culturas y los consumos culturales, plantea un reto múltiple a la educación e incrementa la relevancia de la investigación sociocultural.

James Carey, reconocido como uno de los fundadores en Estados Unidos de los estudios culturales, distinguía ya en 1975 dos concepciones alternas de comunicación, ambas derivadas del pensamiento religioso, que él llamó la visión "transmisional" y la visión "ritual". La primera, más común en las culturas industrializadas, define la comunicación como sinónimo de "impartir", "emitir", "transmitir" o "dar información a otros", mientras que la segunda, ancestral, lo hace en términos de "compartir", "participación", "asociación", "camaradería" y "la posesión común de una fe". El núcleo de la primera visión es la transmisión de señales o mensajes a distancia con propósitos de control (en su origen, religioso o teológico, y después económico y político) y está asociado al transporte, a la extensión de los mensajes en el espacio. Por su parte, la visión ritual se orienta hacia el 
mantenimiento de la sociedad en el tiempo. Su núcleo está en la expresión o representación de creencias compartidas, en la "comunión", en la cultura (Carey, 1989).

Si sostenemos, entonces, un concepto de comunicación basado en la producción y circulación social del sentido, como lo hacen para el estudio de los medios en la sociedad contemporánea, aunque desde perspectivas teóricas diversas, el danés Klaus Bruhn Jensen (1995) y el británico John B. Thompson (1995) entre otros, es posible precisar y rearticular los conceptos de tecnología y cultura con que trabajamos sobre esta confluencia de la educación y la comunicación. Porque adoptar y desarrollar un concepto de comunicación como práctica de producción social de sentido, exige rearticular un concepto amplio de tecnología que evite que ésta sea considerada sólo como algo "externo" o ajeno a las prácticas socioculturales. La tradicional oposición "tecnología contra cultura" tendría así menor oportunidad para operar como obstáculo en la indagación de las múltiples dimensiones que han puesto en evidencia los "nuevos medios" o los "nuevos ambientes" telemáticos.

En pocas palabras, rearticular los procesos subjetivos e intersubjetivos de significación, a través de los esquemas perceptuales e interpretativos que en cada sector cultural median las relaciones posibles con las estructuras y los sistemas objetivos de procesamiento y difusión de la información, es una clave que, además de restituir la complejidad de los procesos socioculturales en los modelos de comunicación, puede servir para enfatizar la agencia o acción transformadora implícita en las prácticas cotidianas reflexivas, es decir, en la interacción material y simbólica entre sujetos concretamente situados, que supone la recurrencia por parte de ellos tanto a sistemas informacionales como a sistemas de significación, cuya competente mediación a su vez determina la producción y reproducción del sentido: el de las prácticas socioculturales de referencia y el de la comunicación misma. De esta manera, también, desplazando el foco del análisis comunicativo de los medios y los mensajes a los sujetos sociales y los procesos de producción del sentido, podrá abordarse la educación como ejercicio práctico de la reflexividad comunicativa, e investigarse sistemáticamente, por ejemplo, los usos de Internet.

Esta propuesta, que desemboca en el desarrollo, más que de una teoría, de una metodología comunicacional, supone que la búsqueda de métodos y de categorías de análisis para investigar los usos comunicacionales y educativos de Internet bien puede contribuir a realizar las posibilidades democratizadoras que promete la incorporación a la "red de redes", a pesar de su conversión aparentemente definitiva en un medio comercial y de la prevalencia indudable de un "espíritu" privatizador, además, por supuesto, de las disparidades abismales de acceso que no sólo caracterizan aún a Internet en escala global, sino incluso dentro de los propios países "desarrollados".

En muy apretada síntesis, si se caracteriza de entrada a Internet como un hipermedio de comunicación, la investigación sociocultural de sus usos actuales y potenciales, muy concretos, puede comenzar a construirse a partir de la triple dimensión de las funciones informativa, comunicativa y difusiva, en relación con, al menos, los siguientes cuatro recursos: infraestructura, códigos, hábitos y representaciones de un grupo o tipo de usuarios determinados. Este último factor puede ser el punto de partida para una reconstrucción etnográfica de las competencias comunicativas empleadas por los usuarios, ya que es en las representaciones de los sujetos donde se puede observar de manera más inmediata la apropiación construida del recurso y los esquemas operativos de la actividad (sea ésta el entretenimiento, el trabajo, la socialización, el aprendizaje, etc.). 
En otras palabras, la exploración sociocultural de los usos comunicativos de Internet puede comenzar de la manera más productiva y sistemática por el análisis de la doxa, o discurso cotidiano de los sujetos sobre el objeto, siguiendo el modelo de la hermenéutica profunda de John B. Thompson (1993), para después continuar con la aplicación de instrumentos para el análisis sociohistórico (escenarios espacio-temporales y campos de interacción), como un cuestionario y entrevistas informales, presenciales y vía correo electrónico, y la aplicación de instrumentos para el análisis de discurso (descripción de prácticas, argumentación) y el análisis formal (productos de información, comunicación, difusión, en Internet).

A través de la "interpretación/reinterpretación" de los resultados empíricos de estos análisis, se podría concluir con la elaboración de un modelo heurístico que articule, mediante la categoría de usos, las mediaciones entre un sistema tecnológico y un grupo bien definido de sujetos, como producto de la exploración de las condiciones socioculturales, institucionales e individuales de apropiación diferencial de Internet como recurso de información, comunicación y difusión en la actividad cotidiana. En una segunda instancia, y sobre esta base, podrán reconstruirse los procesos de estructuración/reestructuración del pensamiento inducidos por el uso apropiado de los recursos de Internet, pues es claro que estos procesos no pueden considerarse efectos inmediatos "de la tecnología", sino en todo caso productos necesarios de la interacción de los sujetos con sus recursos, en el sentido más preciso del término "aprendizaje".

Finalmente, la exploración de la interacción sociocultural mediada por los recursos de la interactividad digital, que va haciéndose cada vez más presente en Internet conforme los usuarios ajustan sus estructuras de pensamiento y de acción a las posibilidades abiertas por el desarrollo tecnológico y la creatividad de sus aplicaciones comunicativas, abre un horizonte de comprensión del carácter constitutivo de la comunicación en la sociedad y la cultura, que los llamados "medios masivos" limitan y oscurecen. Podrá, entonces, recuperarse quizá el sentido del proyecto de Raymond Williams, quien al fundar los estudios culturales británicos en los años sesenta, había reformulado la relación conceptual entre la comunicación y la educación con la cultura y la sociedad de esta manera:

La comunicación comienza en la lucha por aprender y por describir. Para empezar este proceso en nuestras mentes y hacer pasar sus resultados a otros, dependemos de ciertos modelos de comunicación, ciertas reglas o convenciones a través de las cuales podemos establecer el contacto. Podemos cambiar estos modelos cuando se vuelven inadecuados o podemos modificarlos y extenderlos. Nuestros esfuerzos para hacer eso, y para usar los modelos existentes con éxito, se llevan una gran parte de nuestra energía vital... Más aún, muchos de nuestros modelos de comunicación se convierten, en sí, en instituciones sociales. Ciertas actitudes hacia otros, ciertas formas de expresión, ciertos tonos y estilos se incorporan en instituciones que tienen entonces un gran poder de efecto social... Estos supuestos discutibles frecuentemente están incorporados en instituciones sólidas y prácticas, que entonces enseñan los modelos que las originaron (Williams, 1966: 19-20).

Si la comunicación tiene, en la práctica, esa capacidad institucionalizadora, ese potencial de estructuración sociocultural, esa posibilidad de modelar las identidades y las interacciones sociales o, en una palabra, ese poder educativo, como se ha ido reconociendo paulatinamente, es de la mayor importancia comprender cómo es que esa estructuración se realiza y cómo puede intervenirse en ese proceso histórico multidimensional de construcción social de la realidad. 
A principios del siglo XXI hay un intenso debate intelectual en todas partes, y una tensión pública creciente, en cuanto a la reconfiguración histórica del mundo una vez terminada la guerra fría y roto el precario equilibrio que se había generado después de la segunda guerra mundial. En el contexto de esta transición del orden mundial, se habla de globalización, de pensamiento único, de fin de la historia, de libre comercio, de bloques económicos, de mercados regionales, de crisis o de conciencia ecológica, de reconfiguración de identidades, de individualismo, de competencia y supervivencia del más apto, de desintegración familiar y de nuevos regímenes de socialidad, de terrorismo y guerra preventiva, de fundamentalismos, de exclusión y exterminio, de riesgos, de revolución tecnológica ... y de comunicación.

Pero "comunicación" quiere decir tantas cosas distintas y hasta opuestas, que ya no significa casi nada. Es un lugar común que está presente en prácticamente cualquier discurso social. Así, no sirve para nada, o para casi nada, sino para ocultar y disfrazar prácticas e intereses cada vez más polarizados, intolerantes y violentos. "Comunicación" es lo que hacen las industrias mediáticas cuando difunden noticias y editoriales, anuncios comerciales y entretenimiento; lo que hacen los partidos políticos y gobiernos cuando buscan inducir el voto o la opinión favorable a sus figuras, más que a sus propuestas; lo que hacen los movimientos sociales cuando denuncian el orden globalizante y globalizan el malestar históricamente acumulado, sea en Chiapas, sea en cualquier otro lugar del mundo; es lo que hacen las empresas industriales y comerciales para "posicionarse", como se dice en el argot, en sus mercados, pero también en la conciencia de sus empleados; es lo que hace cualquiera que habla por celular o se conecta por chat, con cualquiera, para hablar de cualquier cosa, etcétera.

Algunos sostenemos hoy, con mayor convicción que antes, que el mundo social y la comunicación no son ni deben ser así, que hay algo más sólido detrás de lo que Castells llama "la cultura de la virtualidad real" $(1999, \mathrm{I})$. Y que si el mundo social requiere de transformaciones radicales es tarea de los universitarios aportar a los agentes de esas transformaciones las explicaciones y orientaciones críticas indispensables para la construcción y ejecución de proyectos sociales apropiados, es decir, no impuestos autoritariamente por el poder mediante la "aplicación" de técnicas o saberes de alguna forma de "ingeniería social". Y la primera condición para la generación de tal aporte es el reconocimiento de que esa capacidad de interpretación del sentido de la realidad social y de elaboración colectiva de proyectos de futuro la tienen ya, de entrada, los agentes sociales. Sobre lo que hay que actuar es sobre las posibilidades de que esas interpretaciones divergentes se compartan, se comuniquen, se construyan conjuntamente. Y esa es y tendrá que seguir siendo la tarea principal de la educación.

Eso implica pensar y asumir la comunicación y la educación no como técnicas instrumentales de manipulación de medios y mensajes, como transmisión de lo que producen unos y consumen otros, sino como una relación social constitutiva, como el mecanismo omnipresente que permite construir los consensos, pero también los desacuerdos, no la unanimidad, sino la diversidad respetuosa y mutuamente comprensiva, la acción social que articula proyectos y sujetos, estructuras y prácticas, que produce y desarrolla una nueva ecología cognitiva y comunicativa. Como lo afirma John Durham Peters (1999: 6): "Comunicación es una rica maraña de hebras intelectuales y culturales que codifica las confrontaciones de nuestro tiempo consigo mismo. Comprender la comunicación es comprender mucho más". 


\section{Referencias bibliográficas}

Carey, James. Communication as Culture. Essays on Media and Society. Nueva York y Londres: Routledge, 1989.

Castells, Manuel. La era de la información. Economía, sociedad y cultura. México: Fondo de Cultura Económica, 1999.

Glander, Timothy. Origins of Mass Communications Research during the American Cold War. Educational effects and contemporary implications. Nueva Jersey: Lawrence Erlbaum Associates, 2000.

Jensen, Klaus Bruhn. The Social Semiotics of Mass Communication. Londres: Sage, 1995.

Krippendorff, Klaus. "The past of communication's hoped-for future", en The Future of the Field, Journal of Communication. Estados Unidos, Vol. 43 N 3, 1993.

Levy, Pierre. As tecnologias da Inteligência. O futuro do pensamento na era da informática. São Paulo: Editora 34, 1993.

Martín-Barbero, Jesús. La educación desde la comunicación. Buenos Aires: Norma (Enciclopedia Latinoamericana de Sociocultura y Comunicación), 2002a.

. Oficio de Cartógrafo. Travesías latinoamericanas de la comunicación en la cultura. Santiago de Chile: Fondo de Cultura Económica, 2002b.

Peters, John Durham. Speaking into the Air. A history of the idea of communication. Chicago: The University of Chicago Press, 1999.

Piaget, Jean. Psicología de la Inteligencia. Buenos Aires: Psiqué, 1971.

Shiller, Dan. Digital Capitalism: Networking the Global Market System. Cambridge: MIT Press, 1999.

Soares, Ismar de Oliveira. "Comunicação/Educação: a emergencia de um novo campo; o perfil de seus profissionais", en Contato $\mathrm{N}^{\circ}$ 2. Brasilia, 1999, pp.19-74.

Thompson, John B. Ideología y Cultura Moderna. México: UAM Xochimilco, 1993. University Press, 1995.

The Media and Modernity. A social theory of the Media. California: Stanford

Williams, Raymond. Communications. Londres: Chatto and Windus, 1966. 